\title{
ON THE DOUBLE NÖRLUND SUMMABILITY OF DOUBLE FOURIER SERIES
}

\author{
YASUO OKUYAMA AND IKUKO MIYAMOTO
}

\begin{abstract}
We extend Rajagopal's theorem [12] to a theorem on the double Nörlund summability of double Fourier series, from which various known results are deduced.
\end{abstract}

1. Let $\left\{p_{n}^{(r)}\right\}(r=1,2)$ be two sequences of constants and let

$$
P_{n}^{(r)}=\sum_{k=0}^{n} p_{k}^{(r)} \neq 0 .
$$

The double series $\sum a_{m n}$ with the sequence of partial sum $\left\{s_{m n}\right\}$ is said to be summable by double Nörlund method, or summable $\left(N, p_{m}^{(1)}, p_{n}^{(2)}\right)$ if $t_{m n}$ tends to a limit as $(m, n) \rightarrow \infty$, where the double Nörlund mean $t_{m n}$ is defined by

$$
t_{m n}=\frac{1}{P_{m}^{(1)} P_{n}^{(2)}} \sum_{i=0}^{m} \sum_{k=0}^{n} p_{m-i}^{(1)} p_{n-k}^{(2)} s_{i k}
$$

(see Herriot [4]). In the special case in which $p_{m}^{(1)}=p_{n}^{(2)}=1$ or $p_{m}^{(1)}=p_{n}^{(2)}=\frac{1}{(n+1)}$, the summability $\left(N, p_{m}^{(1)}, p_{n}^{(2)}\right)$ is the same as the summability $(C, 1,1)$ or the summability $(H, 1,1)$, respectively.

Suppose that $f(u, v)$ is integrable $(L)$ over the square $Q(-\pi, \pi ;-\pi, \pi)$ and is periodic with period $2 \pi$ in each variable.

The double Fourier series of function $f(u, v)$ is

$$
\begin{aligned}
& \sum_{m=0}^{\infty} \sum_{n=0}^{\infty} \lambda_{m n}\left[a_{m n} \cos m u \cos n v+b_{m n} \sin m u \cos n v\right. \\
& \left.+c_{m n} \cos m u \sin n v+d_{m n} \sin m u \sin n v\right] \\
= & \sum_{m=0}^{\infty} \sum_{n=0}^{\infty} \lambda_{m n} A_{m n}(u, v),
\end{aligned}
$$

Received October 29, 1994.

1991 Mathematics Subject Classification. Primary 42B05, 42B08.

Key words and phrases. Double Fourier series, double Nörlund summability. 


$$
\left.\begin{array}{ll}
\lambda_{00}=\frac{1}{4} ; & \\
\lambda_{m 0} \\
\lambda_{0 n} \\
\lambda_{m n}
\end{array}\right\}=\begin{array}{ll}
\frac{1}{2} & \text { for } m>0 \\
\frac{1}{2} & \text { for } n>0 \\
1 & \text { for } m>0, n>0
\end{array}
$$

and

$$
a_{m n}=\frac{1}{\pi^{2}} \iint_{Q} f(u, v) \cos m u \cos n v d u d v
$$

and three other similar expressions for $b_{m n}, c_{m n}$ and $d_{m n}$.

We write

$$
\begin{gathered}
\phi(u, v)=\frac{1}{4}[f(x+u, y+v)+f(x+u, y-v)+f(x-u, y+v)+f(x-u, y-v)-4 f(x, y)] \\
\Phi(u, v)=\int_{0}^{u} \int_{0}^{v}|\phi(s, t)| d s d t \\
\Phi_{1}(u, t)=\int_{0}^{u}|\phi(s, t)| d s \\
\Phi_{2}(s, v)=\int_{0}^{v}|\phi(s, t)| d t
\end{gathered}
$$

and for $r=1,2$

$$
K_{m}^{(r)}(u)=\sum_{k=0}^{m} p_{k}^{(r)} \frac{\sin \left(m-k+\frac{1}{2}\right) u}{\sin \frac{1}{2} u}
$$

2. Let $f(t)$ be a periodic finite-valued function with $2 \pi$ and integrable $(L)$ over $(-\pi, \pi)$. We write

$$
\begin{gathered}
\phi(t)=\frac{1}{2}\{f(x+t)+f(x-t)-2 f(x)\} ; \\
\Phi(t)=\int_{0}^{t}|\phi(u)| d u .
\end{gathered}
$$

Rajagopal [12] previously proved the following nice theorem on the Nörlund summability of Fourier series.

Theorem A. Let a function $p(t)$ be monotone nonincreasing and positive for $t \geq 0$. Let $p_{n}=p(n)$ and let

$$
P(t) \equiv \int_{0}^{t} p(u) d u \rightarrow \infty, \quad \text { as } \quad t \rightarrow \infty .
$$

If, for some fixed $\delta, 0<\delta<1$,

$$
\int_{\pi / n}^{\delta} \Phi(t)\left|\frac{d}{d t} \frac{P\left(\frac{\pi}{t}\right)}{t}\right| d t=o\left(P_{n}\right), \quad \text { as } \quad n \rightarrow \infty,
$$


then the Fourier series of function $f(t)$ is summable $\left(N, p_{n}\right)$ to $f(t)$, at the point $t=x$.

Theorem A contains various results due to Hardy [3], Hirokawa [6], Hirokawa and Kayashima [7], Pati [11], Siddiqi [14] and Singh [15,16].

The purpose of this paper is to extend Theorem A to a theorem on the double Nörlund summability of double Fourier series.

Dealing with the harmonic summability of double Fourier series, Sharma [13] proved the following theorem.

Theorem B. If the conditions

$$
\begin{gathered}
\Phi(u, v)=o(u v / \log 1 / u \log 1 / v), \\
\int_{0}^{\pi} \Phi_{1}(u, t) d t=O(u / \log 1 / u)
\end{gathered}
$$

and

$$
\int_{0}^{\pi} \Phi_{2}(s, v) d s=O(v / \log 1 / v)
$$

hold, then the double Fourier series of function $f(u, v)$ is summable $(H, 1,1)$ to $f(u, v)$, at the point $(u, v)=(x, y)$.

This theorem is a generalization of the theorem due to Hille and Tamarkin [5] for double Fourier series and also is analogous to the theorem of Chow [1] for summability $(C, 1,1)$ of the double Fourier series.

Generalizing Theorem B, Mishra [10] proved the following theorem.

Theorem C. Let a function $P^{(r)}(t)(r=1,2)$ be tending to $\infty$ with $t$ and $a$ function $p^{(r)}(t)(r=1,2)$ be monotonic decreasing and strictly positive for $t \geq 0$, such that

$$
P^{(r)}(t)=\int_{0}^{t} p^{(r)}(x) d x, \quad p^{(r)}(n)=p_{n}^{(r)}
$$

If the conditions

$$
\begin{gathered}
\Phi(u, v)=o\left(u v / \Psi^{(1)}(1 / u) \Psi^{(2)}(1 / v)\right), \\
\int_{0}^{\pi} \Phi_{1}(u, t) d t=O\left(u / \Psi^{(1)}(1 / u)\right)
\end{gathered}
$$

and

$$
\int_{0}^{\pi} \Phi_{2}(s, v) d s=O\left(v / \Psi^{(2)}(1 / v)\right)
$$

hold, then the double Fourier series of function $f(u, v)$ is summable $\left(N, p_{m}^{(1)}, p_{n}^{(2)}\right)$ to $f(u, v)$, at the point $(u, v)=(x, y)$, where $\Psi^{(r)}(t)(r=1,2)$ is a positive nondecreasing function with $t$ such that

$$
\int_{1}^{n} \frac{P^{(r)}(x)}{x \Psi^{(r)}(x)} d x=O\left(P_{n}^{(r)}\right) .
$$


If we put $p_{n}^{(r)}=1 /(n+1)$ and $\Psi^{(r)}(x)=\log x$ in Theorem C, we can obtain Theorem $\mathrm{B}$ from Theorem $\mathrm{C}$.

Though the reviewer ([MR] 87f:42034) pointed aut that there appear to be errors in the proof of Theorem C, we think that Theorem $C$ is essentially true.

Now we generalize these Theorems B and $\mathrm{C}$ in the following form.

Theorem. Let a function $P^{(r)}(t)(r=1,2)$ be tending to $\infty$ with $t$ and a function $p^{(r)}(t)(r=1,2)$ be monotonic decreasing and strictly positive for $t \geq 0$, such that

$$
P^{(r)}(t)=\int_{0}^{t} p^{(r)}(x) d x, \quad p^{(r)}(n)=p_{n}^{(r)} .
$$

If the conditions

$$
\begin{gathered}
\int_{1 / m}^{\delta} \int_{1 / n}^{\tau} \Phi(u, v)\left|\frac{d}{d u} \frac{P^{(1)}(1 / u)}{u}\right|\left|\frac{d}{d v} \frac{P^{(2)}(1 / v)}{v}\right| d u d v=o\left(P_{m}^{(1)} P_{n}^{(2)}\right), \\
\text { as }(m, n) \rightarrow \infty \\
\int_{0}^{\pi} d t \int_{1 / m}^{\delta} \Phi_{1}(u, t)\left|\frac{d}{d u} \frac{P^{(1)}(1 / u)}{u}\right| d u=O\left(P_{m}^{(1)}\right), \quad \text { as } m \rightarrow \infty
\end{gathered}
$$

and

$$
\int_{0}^{\pi} d s \int_{1 / n}^{\tau} \Phi_{2}(s, v)\left|\frac{d}{d v} \frac{P^{(2)}(1 / v)}{v}\right| d v=O\left(P_{n}^{(2)}\right), \quad \text { as } n \rightarrow \infty
$$

hold for $0<\delta, \tau<\pi$, then the double Fourier series of function $f(u, v)$ is summable $\left(N, p_{m}^{(1)}, p_{n}^{(2)}\right)$ to $f(u, v)$, at the point $(u, v)=(x, y)$.

If the condition (2.4) holds, then we have by (2.7)

$$
\begin{aligned}
& \int_{1 / m}^{\delta} \int_{1 / n}^{\tau} \Phi(u, v)\left|\frac{d}{d u} \frac{P^{(1)}(1 / u)}{u} \| \frac{d}{d v} \frac{P^{(2)}(1 / v)}{v}\right| d u d v \\
= & o\left(\int_{1 / m}^{\delta} \int_{1 / n}^{\tau} \frac{u v}{\Psi^{(1)}(1 / u) \Psi^{(2)}(1 / v)} \cdot \frac{P^{(1)}(1 / u)}{u^{2}} \cdot \frac{P^{2}(1 / v)}{v^{2}} d u d v\right) \\
= & o\left(\int_{1 / m}^{\delta} \frac{P^{(1)}(1 / u)}{u \Psi^{(1)}(1 / u)} d u \int_{1 / n}^{\tau} \frac{P^{(2)}(1 / v)}{v \Psi^{(2)}(1 / v)} d v\right) \\
= & o\left(\int_{1 / \delta}^{m} \frac{P^{(1)}(x)}{x \Psi^{(1)}(x)} d x \int_{1 / \tau}^{n} \frac{P^{(2)}(y)}{y \Psi^{(2)}(y)} d y\right) \\
= & o\left(P_{m}^{(1)} P_{n}^{(2)}\right)
\end{aligned}
$$

by virture of the fact that $\frac{d}{d u}\left(\frac{P^{(r)}(1 / u)}{u}\right)=O\left(\frac{P^{(r)}(1 / u)}{u^{2}}\right)$. 
Similarly, we have by the conditions (2.5) and (2.7)

$$
\begin{aligned}
& \left.\int_{0}^{\pi} d t \int_{1 / m}^{\delta} \Phi_{1}(u, t) \mid \frac{d}{d u} \frac{P^{(1)}(1 / u)}{u}\right) \mid d u \\
= & \int_{1 / m}^{\delta} \int_{0}^{\pi} \Phi_{1}(u, t) d t\left|\frac{d}{d u} \frac{P^{(1)}(1 / u)}{u}\right| d u \\
= & O\left(\int_{1 / m}^{\delta} \frac{u}{\Psi^{(1)}(1 / u)} \cdot \frac{P^{(1)}(1 / u)}{u^{2}} d u\right) \\
= & O\left(\int_{1 / \delta}^{m} \frac{P^{(1)}(x)}{x \Psi^{(1)}(x)} d x\right) \\
= & O\left(P_{m}^{(1)}\right) .
\end{aligned}
$$

Also, the conditions (2.6) and (2.7) imply the condition (2.10). Thus we see that our theorem is a generalization of Theorems B and C.

3. We need some lemmas for the proof of our Theorem.

Lemma 1 [9]. If a sequence $\left\{p_{n}^{(r)}\right\}(r=1,2)$ is nonnegative and nonincreasing, then we have

$$
\left|\sum_{k=0}^{n} p_{k}^{(r)} \sin \left(n-k+\frac{1}{2}\right) u\right| \leq C P^{(r)}(1 / u),
$$

where $C$ is a positive constant.

Lemma 2. ( $i)$ The condition (2.8) implies the conditon $\Phi(u, v)=o(u v)$. (ii) The condition (2.9) or (2.10) implies the condition $\int_{0}^{\pi} \Phi_{1}(1 / m, t) d t=O(1 / m)$ or $\int_{0}^{\pi} \Phi_{2}(s, 1 / n) d s=O(1 / n)$, respectively.

Proof. (i) By the condition (2.8), we have

$$
\begin{aligned}
o\left(P_{m}^{(1)} P_{n}^{(2)}\right) & =\int_{1 / m}^{\delta} \int_{1 / n}^{\tau} \Phi(u, v)\left|\frac{d}{d u} \frac{P^{(1)}(1 / u)}{u}\right|\left|\frac{d}{d v} \frac{P^{(2)}(1 / v)}{v}\right| d u d v \\
& \geq \Phi(1 / m, 1 / n) \int_{1 / m}^{\delta}\left|\frac{d}{d u} \frac{P(1 / u)}{u}\right| d u \int_{1 / n}^{\pi}\left|\frac{d}{d v} \frac{P^{(2)}(1 / v)}{v}\right| d v \\
& =\Phi(1 / m, 1 / n)\left\{-\int_{1 / m}^{\delta} \frac{d}{d u} \frac{P^{(1)}(1 / u)}{u} d u\right\}\left\{-\int_{1 / n}^{\tau} \frac{d}{d v} \frac{P^{(2)}(1 / v)}{v} d v\right\} \\
& =\Phi(1 / m, 1 / n)\left\{m P^{(1)}(m)-\frac{1}{\delta} P^{(1)}(1 / \delta)\right\}\left\{n P^{(2)}(n)-\frac{1}{\tau} P^{(2)}(1 / \tau)\right\} \\
& \sim m n \Phi(1 / m, 1 / n) P^{(1)}(m) P^{(2)}(n) .
\end{aligned}
$$

Hence we have $\Phi(1 / m, 1 / n)=o(1 / m n)$. Since $\Phi\left(u_{1}, v_{1}\right) \leq \Phi\left(u_{2}, v_{2}\right)$ for $u_{1} \leq u_{2}$ and $v_{1} \leq v_{2}$, we obtain $\Phi(u, v)=o(u v)$. 
(ii). By the condition (2.10), we have

$$
\begin{aligned}
O\left(P_{n}^{(2)}\right) & =\int_{0}^{\pi} d s \int_{1 / n}^{\tau} \Phi_{2}(s, v)\left|\frac{d}{d v} \frac{P^{(2)}(1 / v)}{v}\right| d v \\
& =\int_{1 / n}^{\tau}\left|\frac{d}{d v} \frac{P^{(2)}(1 / v)}{v}\right| d v \int_{0}^{\pi} \Phi_{2}(s, v) d s \\
& =\int_{1 / n}^{\tau}\left|\frac{d}{d v} \frac{P^{(2)}(1 / r)}{v}\right| d v \int_{0}^{\pi} d s \int_{0}^{v}|\phi(s, t)| d t \\
& \geq \int_{1 / n}^{\tau}\left|\frac{d}{d v} \frac{P^{(2)}(1 / v)}{v}\right| d v \int_{0}^{\pi} d s \int_{0}^{1 / n}|\phi(x, t)| d t \\
& =-\int_{1 / n}^{\tau} \frac{d}{d v} \frac{P^{(2)}(1 / v)}{v} d v \int_{0}^{\pi} \Phi_{2}(s, 1 / n) d s \\
& =\left(n P^{(2)}(n)-\frac{1}{\tau} P^{(2)}(1 / \tau)\right) \int_{0}^{\pi} \Phi_{2}(s, 1 / n) d s \\
& \sim n P^{(2)}(n) \int_{0}^{\pi} \Phi_{2}(s, 1 / n) d s .
\end{aligned}
$$

Thus we have $\int_{0}^{\pi} \Phi_{2}(s, 1 / n) d s=O(1 / n)$. The other case is similarly proved.

4. Proof of Theorem. By (1.1), we have

$$
\begin{aligned}
\pi^{2} t_{m n} & =\frac{1}{P_{m}^{(1)} P_{n}^{(2)}} \int_{0}^{\pi} \int_{0}^{\pi} \phi(s, t) K_{m}^{(1)}(s) K_{n}^{(2)}(t) d s d t \\
& =\frac{1}{P_{m}^{(1)} P_{n}^{(2)}}\left[\int_{0}^{\delta} \int_{0}^{\tau}+\int_{0}^{\delta} \int_{\tau}^{\pi}+\int_{\delta}^{P} \int_{0}^{\tau}+\int_{\delta}^{\pi} \int_{\tau}^{\pi}\right] \phi(s, t) K_{m}^{(1)}(s) K_{n}^{(2)}(t) d s d t \\
& \equiv I_{1}+I_{2}+I_{3}+I_{4}
\end{aligned}
$$

say.

Now let $m^{-1}<\delta<\pi, n^{-1}<\tau<\pi$. Then we obtain

$$
\begin{aligned}
I_{3} \leq & \frac{1}{P_{m}^{(1)} P_{n}^{(2)}} \int_{\delta}^{\pi} \int_{0}^{\tau}\left|\phi(s, t)\left\|K_{m}^{(1)}(s)\right\| K_{n}^{(2)}(t)\right| d s d t \\
= & \frac{1}{P_{m}^{(1)} P_{n}^{(2)}} \int_{\delta}^{\pi}\left|K_{m}^{(1)}(s)\right| d s \int_{0}^{1 / n}\left|\phi(s, t) \| K_{n}^{(2)}(t)\right| d t \\
& \quad+\frac{1}{P_{m}^{(1)} P_{n}^{(2)}} \int_{\delta}^{\pi}\left|K_{m}^{(1)}(s)\right| d s \int_{1 / n}^{\tau}\left|\phi(s, t) \| K_{n}^{(2)}(t)\right| d t \\
= & I_{31}+I_{32}
\end{aligned}
$$


say. By Lemmas 1 and 2, we have

$$
\begin{aligned}
I_{31} & =O\left(\frac{1}{P_{m}^{(1)} P_{n}^{(2)}} \int_{\delta}^{\pi} \frac{P^{(1)}(1 / s)}{s} d s \int_{0}^{1 / n}|\phi(s, t)| O\left(n P_{n}^{(2)}\right) d t\right) \\
& =O\left(\frac{n}{P_{m}^{(1)}} \int_{0}^{\pi} \Phi_{2}(s, 1 / n) d s\right) d s \\
& =O\left(\frac{1}{P_{m}^{(1)}}\right) \\
& =O(1), \quad \text { as }(m, n) \rightarrow \infty .
\end{aligned}
$$

Applying Lemma 1 and integrating by parts, we obtain

$$
\begin{aligned}
I_{32} \leq & \frac{1}{P_{m}^{(1)} P_{n}^{(2)}} \int_{\delta}^{\pi}\left|K_{m}^{(1)}(s) d s\right| \int_{1 / n}^{\tau}|\phi(s, t)|\left|K_{n}^{(2)}(t)\right| d t \\
= & O\left[\frac{1}{P_{m}^{(1)} P_{n}^{(2)}} \int_{\delta}^{\pi} \frac{P^{(1)}(1 / s)}{s} d s \int_{1 / n}^{\tau}|\phi(s, t)| \frac{P^{(2)}(1 / t)}{t} d t\right] \\
= & O\left[\frac{1}{P_{m}^{(1)} P_{n}^{(2)}} \int_{\delta}^{\pi} d s\left\{\left[\Phi_{2}(s, t) \frac{P^{(2)}(1 / t)}{t}\right]_{1 / n}^{\tau}-\int_{1 / n}^{\tau} \Phi_{2}(s, t) \frac{d}{d t} \frac{P^{(2)}(1 / t)}{t} d t\right\}\right] \\
= & O\left(\frac{1}{P_{m}^{(1)} P_{n}^{(2)}} \frac{P^{(2)}(1 / \tau)}{\tau} \int_{\delta}^{\pi} \Phi_{2}(s, \tau) d s\right)+O\left(\frac{n}{P_{m}^{(1)}} \int_{\delta}^{\pi} \Phi_{2}(s, 1 / n) d s\right) \\
& +O\left(\frac{1}{P_{m}^{(1)} P_{n}^{(2)}} \int_{\delta}^{\pi} d s \int_{1 / n}^{\tau} \Phi_{2}(s, t)\left|\frac{d}{d t} \frac{P^{(2)}(1 / t)}{t}\right| d t\right) \\
= & O\left(I_{321}+I_{322}+I_{323}\right),
\end{aligned}
$$

say. Clearly we get $I_{321}=o(1)$. By Lemma 2, we have

$$
\begin{aligned}
I_{322} & =\frac{n}{P_{m}^{(1)}} \int_{\delta}^{\pi} \Phi_{2}(s, 1 / n) d s \\
& =O\left(\frac{1}{P_{m}^{(1)}}\right) \\
& =o(1), \quad \text { as }(m, n) \rightarrow \infty .
\end{aligned}
$$

By the condition (2.10), we have

$$
\begin{aligned}
I_{323} & =O\left(\frac{P_{n}^{(2)}}{P_{m}^{(1)} P_{n}^{(2)}}\right) \\
& =O\left(\frac{1}{P_{m}^{(1)}}\right) \\
& =o(1), \quad \text { as }(m, n) \rightarrow \infty .
\end{aligned}
$$

Thus we get $I_{3}=o(1)$. Similarly, we get $I_{2}=o(1)$. 

have

Moreover, since $K_{m}^{(1)}(s)$ and $K_{n}^{(2)}(t)$ are bounded on $[\delta, \pi]$ and $[\tau, \pi]$ respectively, we

$$
\begin{aligned}
I_{4} & =O\left(\frac{1}{P_{m}^{(1)} P_{n}^{(2)}} \int_{\delta}^{\pi} \int_{\tau}^{\pi} \mid \phi(s, t)\left\|K_{m}^{(1)}(s)\right\| K_{n}^{(2)}(t) d s d t\right) \\
& =O\left(\frac{1}{P_{m}^{(1)} P_{n}^{(2)}} \int_{\delta}^{\pi} \int_{\tau}^{\pi}|\phi(s, t)| d s d t\right) \\
& =o(1), \quad \text { as }(m, n) \rightarrow \infty
\end{aligned}
$$

Finally we obtain

$$
\begin{aligned}
I_{1} \leq & \frac{1}{P_{m}^{(1)} P_{n}^{(2)}}\left[\int_{0}^{1 / m} \int_{0}^{1 / n}+\int_{1 / m}^{\delta} \int_{0}^{1 / n}+\int_{0}^{1 / m} \int_{1 / n}^{\tau}\right. \\
& \left.+\int_{1 / m}^{\delta} \int_{1 / n}^{\tau}\right]\left|\phi(s, t)\left\|K_{m}^{(1)}(t)\right\| K_{n}^{(2)}(s)\right| d s d t \\
= & I_{11}+I_{12}+I_{13}+I_{14},
\end{aligned}
$$

say. By Lemma 2, we have

$$
\begin{aligned}
I_{11} & \leq \frac{1}{P_{m}^{(1)} P_{n}^{(2)}} \int_{0}^{1 / m} \int_{0}^{1 / n}|\phi(s, t)| O\left(m n P_{m}^{(1)} P_{n}^{(2)}\right) d s d t \\
& =O\left(m n \int_{0}^{1 / m} \int_{0}^{1 / n}|\phi(s, t)| d s d t\right) \\
& =o(1), \quad \text { as }(m, n) \rightarrow \infty .
\end{aligned}
$$

Appling Lemma 1 and integrating by parts, we obtain

$$
\begin{aligned}
I_{12} \leq & \frac{1}{P_{m}^{(1)} P_{n}^{(2)}} \int_{0}^{1 / n}\left|K_{n}^{(2)}(t)\right| d t \int_{1 / m}^{\delta}|\phi(s, t)|\left|K_{m}^{(1)}(s)\right| d s \\
= & O\left(\frac{1}{P_{m}^{(1)} P_{n}^{(2)}} \int_{0}^{1 / n}\left(n P_{n}^{(2)}\right) d t \int_{1 / m}^{\delta}|\phi(s, t)| \frac{P^{(1)}(1 / s)}{s} d s\right) \\
= & O\left(\frac{n}{P_{m}^{(1)}} \int_{0}^{1 / n} d t \int_{1 / m}^{\delta}|\phi(s, t)| \frac{P^{(1)}(1 / s)}{s} d s\right) \\
= & O\left(\frac{n}{P_{m}^{(1)}} \int_{0}^{1 / n} d t\left\{\left[\Phi_{1}(s, t) \frac{P^{(1)}(1 / s)}{s}\right]_{1 / m}^{\delta}-\int_{1 / m}^{\delta} \Phi_{1}(s, t) \frac{d}{d s}\left(\frac{P^{(1)}(1 / s)}{s}\right) d s\right\}\right) \\
= & O\left(\frac{n}{P_{m}^{(1)}} \int_{0}^{1 / n} \Phi_{1}(\delta, t) \frac{P^{(1)}(1 / \delta)}{\delta} d t+\frac{n}{P_{m}^{(1)}} \frac{P^{(1)}(m)}{\frac{1}{m}} \int_{0}^{1 / n} \Phi_{1}(1 / m, t) d t\right. \\
& \left.+\frac{n}{P_{m}^{(1)}} \int_{0}^{1 / n} d t \int_{1 / m}^{\delta} \Phi_{1}(s, t)\left|\frac{d}{d s} \frac{P^{(1)}(1 / s)}{s}\right| d s\right) \\
= & O\left(I_{121}+I_{122}+I_{123}\right),
\end{aligned}
$$


say. By Lemma 2, we have

$$
\begin{aligned}
I_{121} & =O\left(\frac{n}{P_{m}} \int_{0}^{1 / n} \Phi_{1}(\delta, t) d t\right) \\
& =O\left(\frac{n}{P_{m}} \Phi(\delta, 1 / n)\right) \\
& =O\left(\frac{n}{P_{m}} o\left(\frac{1}{n}\right)\right) \\
& =o\left(\frac{1}{P_{m}}\right) \\
& =o(1), \quad \text { as }(m, n) \rightarrow \infty
\end{aligned}
$$

and

$$
\begin{aligned}
I_{122} & =O\left(m n \int_{0}^{1 / n} \Phi_{1}(1 / m, t) d t\right) \\
& =O(m n \Phi(1 / m, 1 / n)) \\
& =o(1), \quad \text { as }(m, n) \rightarrow \infty .
\end{aligned}
$$

On the other hand, we have by the conditoin (2.8)

$$
\begin{aligned}
o(1) & =\frac{1}{P_{m}^{(1)} P_{n}^{(2)}} \int_{1 / m}^{\delta} \int_{1 / n}^{\tau} \Phi(u, v)\left|\frac{d}{d u} \frac{P^{(1)}(1 / u)}{u}\right|\left|\frac{d}{d v} \frac{P^{(2)}(1 / v)}{v}\right| d u d v \\
& \geq \frac{1}{P_{m}^{(1)} P_{n}^{(2)}} \int_{1 / m}^{\delta} \Phi(u, 1 / n)\left|\frac{d}{d u} \frac{P^{(1)}(1 / u)}{u}\right| d u \int_{1 / n}^{\tau}\left|\frac{d}{d u} \frac{P^{(2)}(1 / v)}{v}\right| d v \\
& \geq A \frac{n P_{n}^{(2)}}{P_{m}^{(1)} P_{n}^{(2)}} \int_{1 / m}^{\delta} \Phi(u, 1 / n)\left|\frac{d}{d u} \frac{P^{(1)}(1 / u)}{u}\right| d u \\
& \geq A \frac{n}{P_{m}^{(1)}} \int_{0}^{1 / n} d t \int_{1 / m}^{\delta} \Phi_{1}(u, t)\left|\frac{d}{d u} \frac{P^{(1)}(1 / u)}{u}\right| d u \\
& =A I_{123} .
\end{aligned}
$$

Thus we get $I_{123}=o(1)$. Hence we have $I_{12}=o(1)$. Similarly, we have $I_{13}=o(1)$.

By partial integration for double integral $[2,8]$ and Lemma 1 , we have

$$
I_{14} \leq \frac{1}{P_{m}^{(1)} P_{n}^{(2)}} \int_{1 / m}^{\delta} \int_{1 / n}^{\tau}|\phi(s, t)| \frac{P^{(1)}(1 / s)}{s} \frac{P^{(2)}(1 / t)}{t} d t d s
$$




$$
\begin{aligned}
= & \frac{1}{P_{m}^{(1)} P_{n}^{(2)}} \Phi(\delta, \tau) \frac{P^{(1)}(1 / \delta)}{\delta} \frac{P^{(2)}(1 / \tau)}{\tau} \\
& -\frac{1}{P_{m}^{(1)} P_{n}^{(2)}} \frac{P^{(2)}(1 / \tau)}{\tau} \int_{1 / m}^{\delta} \Phi(u, \tau) \frac{d}{d u}\left(\frac{P^{(1)}(1 / u)}{u}\right) d u \\
& -\frac{1}{P_{m}^{(1)} P_{n}^{(2)}} \frac{P^{(1)}(1 / \delta)}{\delta} \int_{1 / n}^{\delta} \Phi(\delta, v) \frac{d}{d v}\left(\frac{P^{(2)}(1 / v)}{v}\right) d v \\
& +\frac{1}{P_{m}^{(1)} P_{n}^{(2)}} \int_{1 / m}^{\delta} \int_{1 / n}^{\tau} \Phi(u, v) \frac{d}{d u}\left(\frac{P^{(1)}(1 / u)}{u}\right) \frac{d}{d v}\left(\frac{P^{(2)}(1 / v)}{v}\right) d u d v \\
= & I_{141}+I_{142}+I_{143}+I_{144},
\end{aligned}
$$

say. Clearly we get $I_{141}=o(1)$. By the condition (2.8), we have $I_{144}=o(1)$. Also, by the condition (2.9), we have

$$
\begin{aligned}
I_{142} & \leq \frac{1}{P_{m}^{(1)} P_{n}^{(2)}} \frac{P^{(2)}(1 / \tau)}{\tau} \int_{1 / m}^{\delta} \Phi(u, \tau)\left|\frac{d}{d u} \frac{P^{(1)}(1 / u)}{u}\right| d u \\
& =O\left(\frac{1}{P_{m}^{(1)} P_{n}^{(2)}} \int_{0}^{\tau} d t \int_{1 / m}^{\delta} \Phi_{1}(u, t)\left|\frac{d}{d u} \frac{P^{(1)}(1 / u)}{u}\right| d u\right) \\
& =O\left(\frac{1}{P_{m}^{(1)} P_{n}^{(2)}} \int_{0}^{\pi} d t \int_{1 / m}^{\delta} \Phi_{1}(u, t)\left|\frac{d}{d u} \frac{P^{(1)}(1 / u)}{u}\right| d u\right) \\
& =O\left(\frac{1}{P_{m}^{(1)} P_{n}^{(2)}} O\left(P_{m}^{(1)}\right)\right) \\
& =O\left(\frac{1}{P_{n}^{(2)}}\right) \\
& =o(1), \quad \text { as }(m, n) \rightarrow \infty .
\end{aligned}
$$

Similarly, we get $I_{143}=o(1)$. Hence we have $I_{14}=o(1)$. Therefore, by the above estimations, our theorem is completely proved.

5. In this section, we deduce some corollaries from our theorem.

Corollary 1. If the condtions

$$
\begin{aligned}
& \Phi(u, v)=o(u v), \\
& \Phi(u, \pi)=O(u)
\end{aligned}
$$

and

$$
\Phi(\pi, v)=O(v)
$$

hold, then the double Fourier series of function $f(u, v)$ is summable $(C, 1,1)$ to $f(u, v)$, at the point $(u, v)=(x, y)$. 
A weaker form of Corollary 1 was proved by Chow [1].

Corollary 2. Let $p^{(r)}(t)$ and $P^{(r)}(t)(\tau=1,2)$ be the functions satisfying the same hypothesis of Theorem such that

$$
\log n=O\left(P_{n}^{(r)}\right)(r=1,2) .
$$

If the conditions

$$
\begin{gathered}
\Phi(u, v)=o\left(u v / P^{(1)}(1 / u) P^{(2)}(1 / v)\right), \\
\Phi(u, \pi)=O\left(u / P^{(1)}(1 / u)\right)
\end{gathered}
$$

and

$$
\Phi(\pi, v)=O\left(v / P^{(2)}(1 / v)\right)
$$

hold, then the double Fourier series of function $(u, v)$ is summable $\left(N, p_{m}^{(1)}, p_{n}^{(2)}\right)$ to $f(u, v)$, at the point $(u, v)=(x, y)$.

This corollary is also deduced from Theorem C.

\section{References}

[1] Y. S. Chow, "On the Cesàro summability of double Fourier series," Tôhoku Math. J., 5(1953), 277-283.

[2] J. J. Gergen, "Convergence criteria for double Fourier series," Trans. Amer. Math. Soc., 35(1933), 29-63.

[3] G. H. Hardy, "On the summability of Fouier series," Proc. London Math. Soc., 12(1913), 365-372.

[4] J. G. Herriot, "The Nörlund summability of double Fouier series," Trans. Amer. Math. Soc., 59(1942), 72-94.

[5] E. Hille and J. D. Tamarkin, "On the summability of Fouier series," Trans. Amer. Math. Soc., 34(1932), 757-783.

[6] H. Hirokawa, "On the Nörlund summability of Fouier series and its conjugate series," Proc. Japan Acad., 44(1968), 449-451.

[7] H. Hirokawa and I. Kayashima, "On a sequence of Fourier coefficients," Proc. Japan Acad., 50(1974), 57-62.

[8] E. W. Hobson, Theory of functions of a real variable, Cambridge, Vol.1, 1927.

[9] L. McFadden, "Absolute Nörlund summability," Duke Math. J., 9(1942), 168-207.

[10] K. N. Mishra, "Summability of double Fourier series by double Nörlund method," Bull. Inst. Math. Acad. Sinica., 13(1985), 289-295.

[11] T. Pati, "A generalization of a theorem of Igengar on the harmonic summability of Fourier series," Indian J. Math., 3(1961), 85-90.

[12] C. T. Rajagopal, "On the Nörlund summability of Fourier series," Proc. Camb. Phil. Soc., 59(1963), 47-53.

[13] P. L. Sharma, "On the harmonic summability of double Fouier series," Proc. Amer. Math. Soc., 91(1958), 979-986.

[14] J. A. Siddiqi, "On the harmonic summability of Fouier series," Proc. Nat. Acad. Sci. India Sect.A., 28(1948), 527-531.

[15] T. Singh, "On Nörlund summability of Fouier series and its conjugate series," Proc. Nat. Inst. Sci. India Part A., 29(1963), 65-73. 
[16] T. Singh, "Nörlund summahbility of Fourier series and its conjugate series," Ann. Mat. Pura Appl., 64(1964), 123-132.

Department of Mathematics, Faculty of Engineering, Shinshu University, Nagano 380, Japan.

Department of Mathematics and Informatics, Faculty of Science, Chiba University, Chiba 263, Japan. 